\title{
The JWST MIRI instrument concept
}

Wright, G. S.; Rieke, G. H.; Colina, L.; van Dishoech, E; Goodson, G; Greene, T; Lagage, P. O.; Karnik, A; Lambros, S. D.; Lemke, D

Total number of authors:

18

Published in:

SPIE

Publication date:

2004

Document Version

Early version, also known as pre-print

Link back to DTU Orbit

Citation (APA):

Wright, G. S., Rieke, G. H., Colina, L., van Dishoech, E., Goodson, G., Greene, T., Lagage, P. O., Karnik, A., Lambros, S. D., Lemke, D., Meixner, M., Nørgaard-Nielsen, H. U., Oloffson, G., Ray, T., Ressier, M., Waelkens, C., Wright, D., \& Zehnder, A. (2004). The JWST MIRI instrument concept. In SPIE

\section{General rights}

Copyright and moral rights for the publications made accessible in the public portal are retained by the authors and/or other copyright owners and it is a condition of accessing publications that users recognise and abide by the legal requirements associated with these rights.

- Users may download and print one copy of any publication from the public portal for the purpose of private study or research.

- You may not further distribute the material or use it for any profit-making activity or commercial gain

- You may freely distribute the URL identifying the publication in the public portal 


\title{
The JWST MIRI instrument concept
}

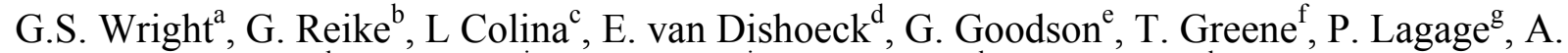

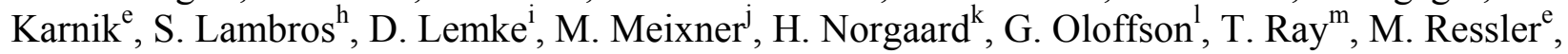
C. Waelkens ${ }^{\mathrm{n}}$, D. Wright ${ }^{\mathrm{o}}$, A.Zhender $^{\mathrm{p}}$

${ }^{a}$ UK Astronomy Technology Centre, Blackford Hill, Edinburgh EH9 3DJ, United Kingdom

${ }^{\mathrm{b}}$ Steward Observatory, University of Arizona, 933 N. Cherry Avenue, Tucson, AZ 85721-0065 U.S.A

${ }^{\mathrm{c} C o n s e j o}$ Superior de Investigaciones Cientificas, Instituto de Estructura de la Materia, C/Serrano 121, 28006-Madrid , Spain

${ }^{\mathrm{d}}$ Sterrewacht Leiden, Postbus 9513, 2300 RA Leiden, The Netherlands

${ }^{\mathrm{e}}$ Jet Propulsion Laboratory, California Institute of Technology, 4800 Oak Grove Drive, Pasadena, CA 91109, USA

${ }^{f}$ Nasa Ames Research Centre, Moffett Field, CA 94035, USA

${ }^{g}$ CEA Saclay, Service d'Astrophysique, Orme de Merisiers, 91191 Gif-sur-Yvette CEDEX France

${ }^{\mathrm{h}}$ NASA Goddard Space Flight Centre, Greenbelt MD 20771, USA

${ }^{\mathrm{i}}$ Max-Planck Institute fur Astronomy, Königstuhl 17, 69117 Heidelberg, Germany

${ }^{j}$ Space Telescope Science Institute, 3700 San Martin Drive, Baltimore, MD 21218, USA

${ }^{k}$ Danish Space Research Institute, Juliane Mariesvej 30, DK-2100 Copenhagen, Denmark

${ }^{1}$ Stockholm Observatory, SCFAB Astronomy, SE-106 91, Sweden

${ }^{\mathrm{m}}$ Dublin Institute for Advanced Studies, 5 Merrion Square, Dublin 2, Ireland

${ }^{n}$ C. Waelkens, Katholieke Univ. Leuven, Institut voor Sterrenkunde, Celestijnenlaan 200B, Heverlee 3001, Beglium

${ }^{\circ}$ EADS-Astrium Ltd, Gunnels Wood Road, Stevenage, SG1 2AS, United Kingdom

${ }^{\mathrm{p}}$ Paul Scherrer Institute, Laboratory for Astrophysics, ODRA 120, 5232 Villigen-PSI, Switzerland

\begin{abstract}
The MIRI is the mid-IR instrument for JWST and provides imaging, coronography and low and medium resolution spectroscopy over the $5-28 \mu \mathrm{m}$ band. In this paper we provide an overview of the key driving requirements and design status.
\end{abstract}

Keywords: JWST, MIRI, mid-Infrared, instrumentation

\section{INTRODUCTION}

The science requirements for MIRI are a key part of the JWST science requirements described in Gardner et al. ${ }^{1}$ and are divided into four key themes:

1. First Light (After the Big Bang)

To find and study the first luminous objects - proto-galaxies, supernovae, black holes

2. Assembly of Galaxies

To study the merging of proto-galaxies, effects of black holes, history of star formation

3. Birth of Stars and Planetary Systems

JWST will study how stars form in dust clouds, and how chemical elements are produced and re-circulated. 
4. Planetary Systems and the Origins of Life

To study the formation of planets and obtain direct observations of other planetary systems, as well as study of the outer solar system.

The science goals indicated briefly above require a versatile mid-infrared instrument with a wide field of view for imaging through broad and narrow band filters, low resolution spectroscopy from 5-10 $\mu$ m, moderate resolution spectroscopy with $\mathrm{R} \sim 3000$, and high dynamic range coronography. MIRI is designed to provide this science capability for a lifetime requirement of 5 years after the completion of commissioning.

MIRI is one of four instruments to be built for JWST. It is being developed as a 50-50 partnership between the USA and Europe. The Jet Propulsion Laboratory is responsible for the US contribution to MIRI and chair the overall management/system teams. The European MIRI contributions are provided by a Nationally Funded Consortium of 21 institutes from 10 countries under the auspices of the European Space Agency.

\section{OVERALL INSTRUMENT CONCEPT}

A schematic of the overall instrument concept is shown in Figure 1.

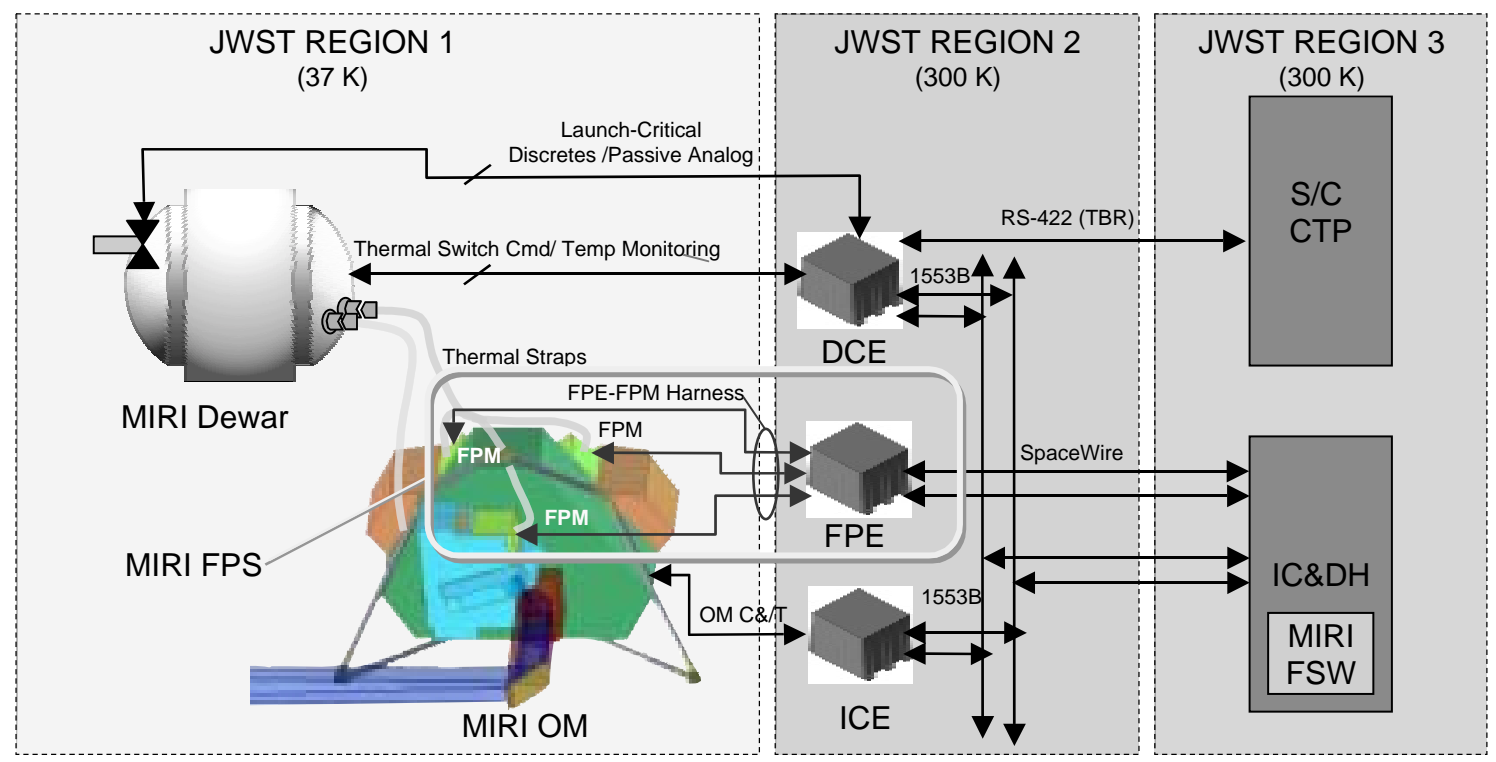

Fig. 1. Schematic of the MIRI System

The instrument has a modular optical design and an iso-thermal all aluminium construction. It is thermally isolated from the ISIM structure by a CFRP hexapod, and cooled by a two stage solid hydrogen Dewar. Optically the instrument is divided into two channels - an imager channel, with one $1 \mathrm{Kx} 1 \mathrm{~K} \mathrm{Si}$ :As detector array and a spectrometer channel, which is further subdivided into two modules each with a $1 \mathrm{kx} 1 \mathrm{~K} \mathrm{Si}$ :As detector array. The Imager Channel provides broad and narrow band filters covering the range 5 to $27 \mu \mathrm{m}$ with $0.11^{\prime}$ " pixels and a $1.3^{\prime} \mathrm{x} 1.7$ ' field of view, coronagraphs at $10.65,11.4,15.5,23 \mu \mathrm{m}$ and incorporates a slit for $\mathrm{R} \sim 100$ spectroscopy from 5 to $10 \mu \mathrm{m}$. The Spectrometer Channel provides medium resolution integral field spectroscopy at $\mathrm{R} \sim 3000$ and covers the full 5 to $28.3 \mu \mathrm{m}$ contiguously in 3 exposures. The spectrograph is subdivided into four image slicer inputs with fields of view on the sky of $3.5 \times 3.5-7 \times 7$ arcsec fov and these provide the inputs to a short and a long wavelength spectrometer. Dedicated electronics boxes are provided for the control of the Dewar, the instrument mechanisms and housekeeping and the focal plane arrays and these interface to the spacecraft and ISIM instrument command and data handling systems as appropriate. 
The overall sensitivity of MIRI has been extensively modeled and is reported in Swinyard et al ${ }^{2}$. Table 1 provides a short summary of the expected performance for imaging and emission line spectroscopy.

\begin{tabular}{||l|l|l||}
\hline $\begin{array}{l}\text { WAVELENGTH } \\
(\mu \mathrm{m})\end{array}$ & TYPE & $10 \sigma, 10,000$ SEC SENSITIVITY \\
\hline 5.6 & Imaging & $0.19 \mathrm{mJy}$ \\
\hline 12.8 & Imaging & $1.4 \mathrm{mJy}$ \\
\hline 25.5 & Imaging & $29 \mathrm{mJy}$ \\
\hline 6.4 & Line Spectroscopy & $1.2 \times 10^{-20} \mathrm{Wm}^{-2}$ \\
\hline 22.5 & Line Spectroscopy & $5.6 \times 10^{-20} \mathrm{Wm}^{-2}$ \\
\hline
\end{tabular}

Table 1. Summary of expected MIRI Sensitivity

|In the following sections we summarise the key features of the MIRI optical, thermal, mechanical design and the focal plane system.

\section{OPTICAL CONCEPT}

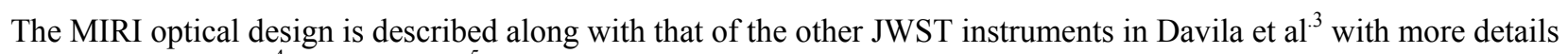
given in Wells et al. ${ }^{4}$ and Lee et al. ${ }^{5}$, so only a short description is provided here. The optical concept splits the instrument into two separate channels operating over the 5 to 28 micron wavelength range, one for Imaging (over a 1.3' $\mathrm{x} 1.7$ ' FOV) and one for medium resolution spectroscopy (up to 8"x8" FOV). In addition, the Imaging channel includes a low resolution $(\mathrm{R} \sim 100)$ spectrometer and a coronograph function. This functional split into two parts was chosen because it was found that it simplified the internal optical interfaces, and the complexity of the layout and of the mechanisms. Both the Imager and Spectrometer channels are fed by common optics from a single pick-off mirror placed close to the Telescope focal plane, and fed also by a common calibration subsystem. The pick-off mirror in front of the JWST OTA focal plane directs the MIRI FOV towards the imager. A small fold mirror adjacent to the imager light path picks off the small (up to $8 \times 8$ arcsec) fov of the spectrometer. A second tilting fold in the spectrometer optical path is used to select either light from the telescope or from the MIRI calibration system.

The Imager module has a combined FOV for the imager and coronagraph/low-resolution spectrometer modes. The coronagraph masks are placed at a fixed location on one edge of the imager field. The light is collimated and, at the pupil image formed by the collimator, a filter wheel holds the imaging filters, Lyot stops and filters for the coronograph and a pair of prisms for the low-resolution 5-10 $\mu \mathrm{m}$ spectroscopy. A camera then images the field (or spectrum) onto the single $1 \mathrm{kx} 1 \mathrm{k}$ detector.

The medium resolution Spectrometer module is split into a Short-Wavelength $(\sim 5-12 \mu \mathrm{m})$ and a Long Wavelength channel $(\sim 12-28 \mu \mathrm{m})$ each covering two of four wavelength quadrants split by dichroics. Each quadrant consists of an Integral Field Unit whose output is collimated and then dispersed by a dedicated first-order diffraction grating. The spectra from pairs of the IFUs are then combined by two cameras onto two $1 \mathrm{k} \mathrm{x} 1 \mathrm{k}$ detectors. The spectrometer optics fall naturally into two subsystems - a Spectrometer Pre-Optics which consists of the system of dichroics and image slicers with associated fold and re-imaging mirrors, and a Spectrometer Main Optics consisting of the gratings and camera systems for the two channels.

The calibration system is an integrating sphere (IS) with blackbody sources for flat-fielding the detector arrays and for wavelength calibration of the spectrometer. The same Integrating Sphere is used for both the imager and spectrometer, the light path to the imager is via a small mirror mounted in the central obscuration at the reimaged pupil.

\section{THE FOCAL PLANE SYSTEM}

In simple terms, the role of the MIRI Focal Plane System (FPS) is to convert infrared photons directed onto the detectors by the MIRI Optics Assembly (OA) into electrons, and transmit the corresponding data to higher-level 
handlers. Specifically, the FPS responds to observation setup commands, produces the clocks and biases necessary to drive the detectors and read-out electronics, receives the analog signals from the detectors, amplifies and digitizes those signals, and transmits the data to the JWST Integrated Science Instrument Module (ISIM) Command and Data Handling System (ICDH). There are three Focal Plane Modules (FPMs), a single Focal Plane Electronics (FPE) unit, and a set of low noise FPE/FPM cryogenic harnesses that connect the FPMs to the FPE.

\subsection{Focal Plane Module Description}

Each Focal Plane Module (FPM) houses a single Sensor Chip Assembly (SCA) containing a 1024 x 1024 Si:As detector array and readout electronics. More details about the SCA can be found in Love et al. ${ }^{6}$ The design of the three FPMs is identical, except for the anti-reflective coatings applied to the detector to maximize the absorption of IR photons within the desired instrument bandpass. This allows a single FPM design to fulfill the needs of the four required MIRI science modes.

The FPM housing locates the front surface of the detector at a focal plane interface provided by the Optics Assembly and provides all necessary thermal and electrical isolation. One FPM is mounted on the imager assembly; one is located on each of the two medium resolution Integral Field Unit (IFU) spectrometer assemblies.

JWST science and functional requirements provide the primary design drivers for the FPS sensitivity and functional performance. These are summarized in Table 2 .

Table 2. MIRI FPS Requirements

\begin{tabular}{|c|c|}
\hline Parameter & $\begin{array}{r}\text { Requirement } \\
\end{array}$ \\
\hline SCA format (FOV) & $1024 \times 1024$ pixels \\
\hline Pixel pitch & 18 to $30 \mu \mathrm{m}$ \\
\hline Detector type (mid-IR bandpass) & Si:As \\
\hline Fill factor & $100 \%$ \\
\hline Number of outputs & Read-out in $\leq 3$ seconds \\
\hline Partial array read & Subarray readout \\
\hline Optical Transmissivity & Maximize in-band signal \\
\hline Total Detector Noise & $<19$ e- (multiply sampled) \\
\hline Dark Current & Part of total noise \\
\hline Read Noise & Part of total noise \\
\hline Quantum Efficiency & $>50 \% @ 5 \mu \mathrm{m}$ \\
\hline Spectral Response & 5 to $27 \mu \mathrm{m}$ \\
\hline Response at $28.2 \mu \mathrm{m}$ & None \\
\hline Well capacity & $>100,000$ e- \\
\hline Pixel operability & $>99 \%$ \\
\hline Bad pixel clustering & TBD \\
\hline Calibration error & $<1 \%$ \\
\hline Linearity & $<0.1 \%$ from $10 \%$ to $80 \%$ of well (after cal.) \\
\hline Pix-to-pix uniformity & $<10 \%$ (before cal.) \\
\hline Detector MTF & $>53 \%$ \\
\hline Active power dissipation & $<1 \mathrm{~mW}$ per SCA \\
\hline Latent and residual images & $<0.1 \%$ (after saturation) \\
\hline Cross-talk & $<1 \%$ \\
\hline
\end{tabular}




\begin{tabular}{|l|l|}
\hline Cosmic Ray defects & Allow integrations up to $4000 \mathrm{sec}$ \\
\hline Radiation Environment & $\begin{array}{l}5 \mathrm{krad}(\mathrm{Si}) \mathrm{TID} \text {, including } 2 \mathrm{xRDM} \text { for } 5.5 \mathrm{yr} . ; \\
\text { pixels } \\
\text { (General JWST requirement is } 42.5 \mathrm{krad}(\mathrm{Si}) \mathrm{TID}, 2.54 \mathrm{~mm} \mathrm{Al}, 5.5 \mathrm{yr} \text {.) }\end{array}$ \\
\hline Detector Placement & Maintain correct 3-D position after cool down \\
\hline Mass & $3 \mathrm{~kg}$ allocation (each), including uncertainty and margin \\
\hline Thermal stability & $10 \mathrm{mK}$ during $1000 \mathrm{sec}$ exposure \\
\hline
\end{tabular}

In addition, the FPMs provide the following functionality:

- Transfer the electrical signals to/from the detector from/to a suitable connector

- Electrically isolate the detector from the surrounding housing

- Provide the mechanical interface for electrical harnesses

- Maintain the detector temperature at the specified operating point

- Provide a high conductance thermal link between the detector and dewar

- Thermally isolate the detector from the surrounding housing

- Reduce the thermal spike presented to the dewar during detector annealing

- $\quad$ Temperature Monitoring

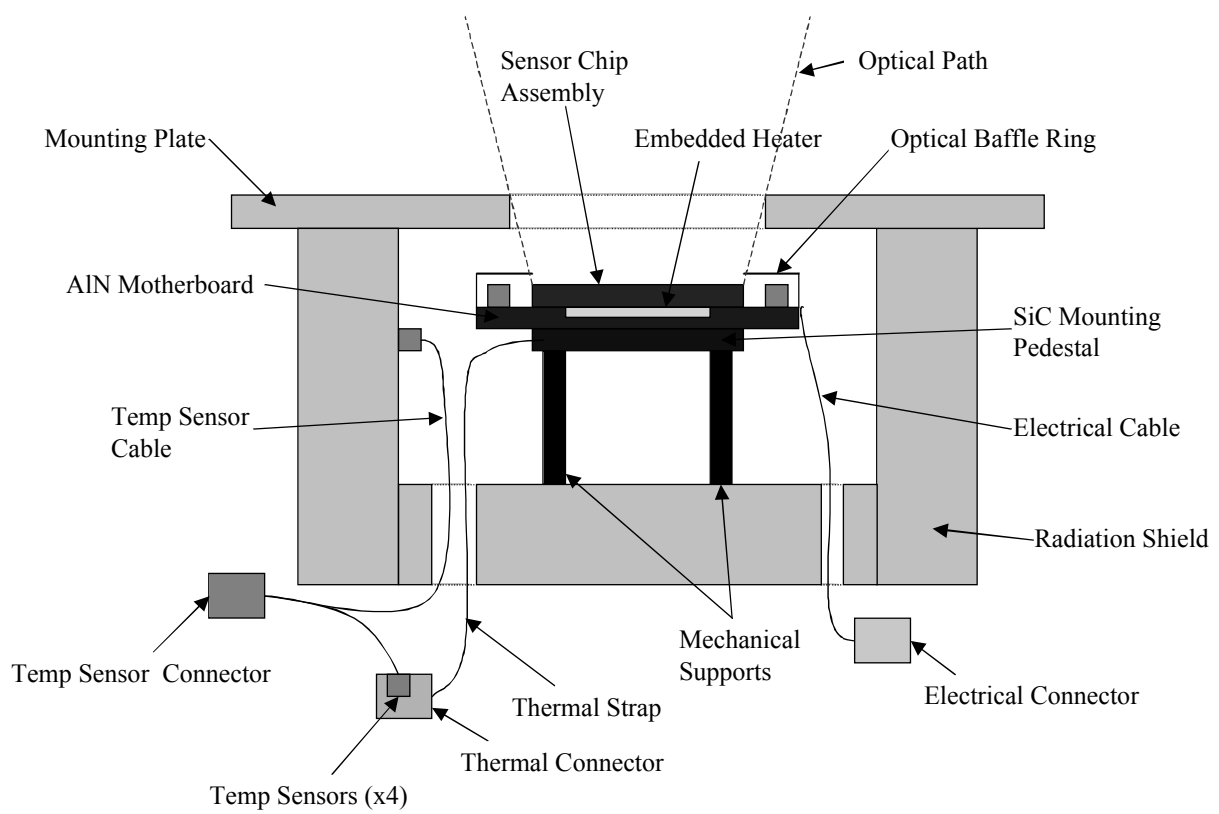

Figure 2. Function Block Diagram of Focal Plane Module

To meet the stated sensitivity, dark current and background must be reduced to a minimum. This will require the detectors to operate at $7.1 \mathrm{~K}$ (End of Life)). All detector and Optical Assembly cooling will be provided by conductive thermal straps connected to the MIRI solid hydrogen dewar. The FPM functional design necessary to support these requirements is shown in the block diagram in Figure 2. An early conceptual mechanical design for the FPMs is shown the illustration in Figure 3. 


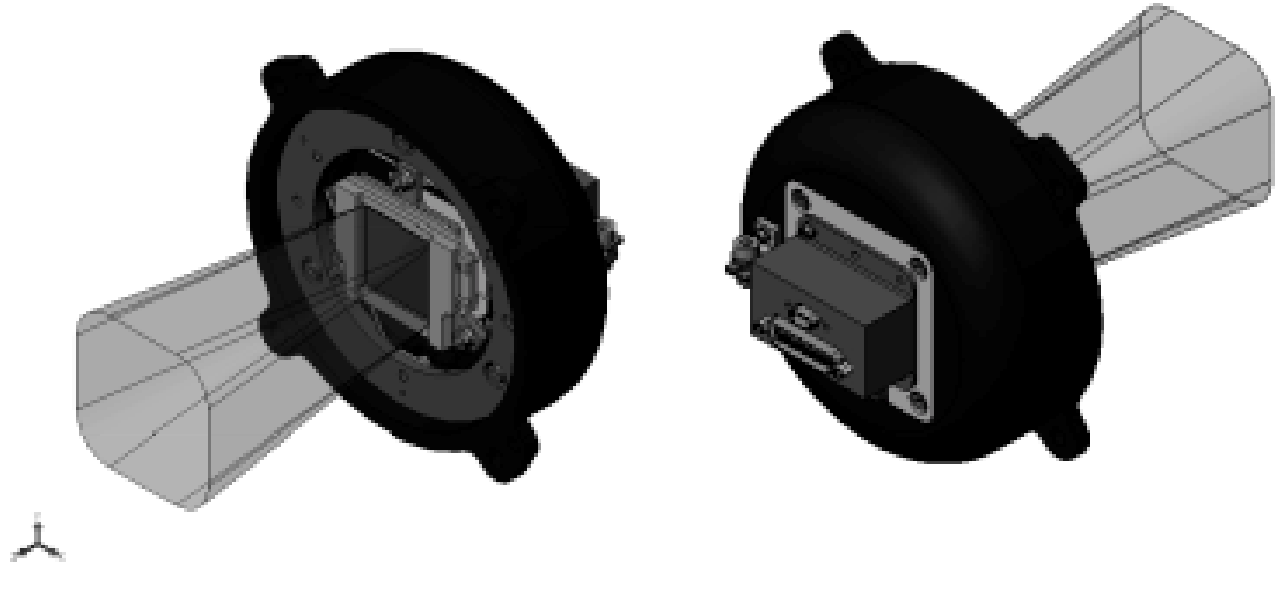

Figure 3. Conceptual FPM Mechanical Illustration

\subsection{Focal Plane Electronics and Harness}

The FPE is a space-qualified electronics box located in a room temperature compartment on the ISIM in Region 2. It is the command and data handling interface for the three FPMs as shown in the block diagram, Figure 4.

It does not posses an on-board programmable processor. Instead, it receives setup commands for the individual FPM SCAs from the MIRI flight software (FSW), which is an instrument-specific application within the JWST ICDH and its

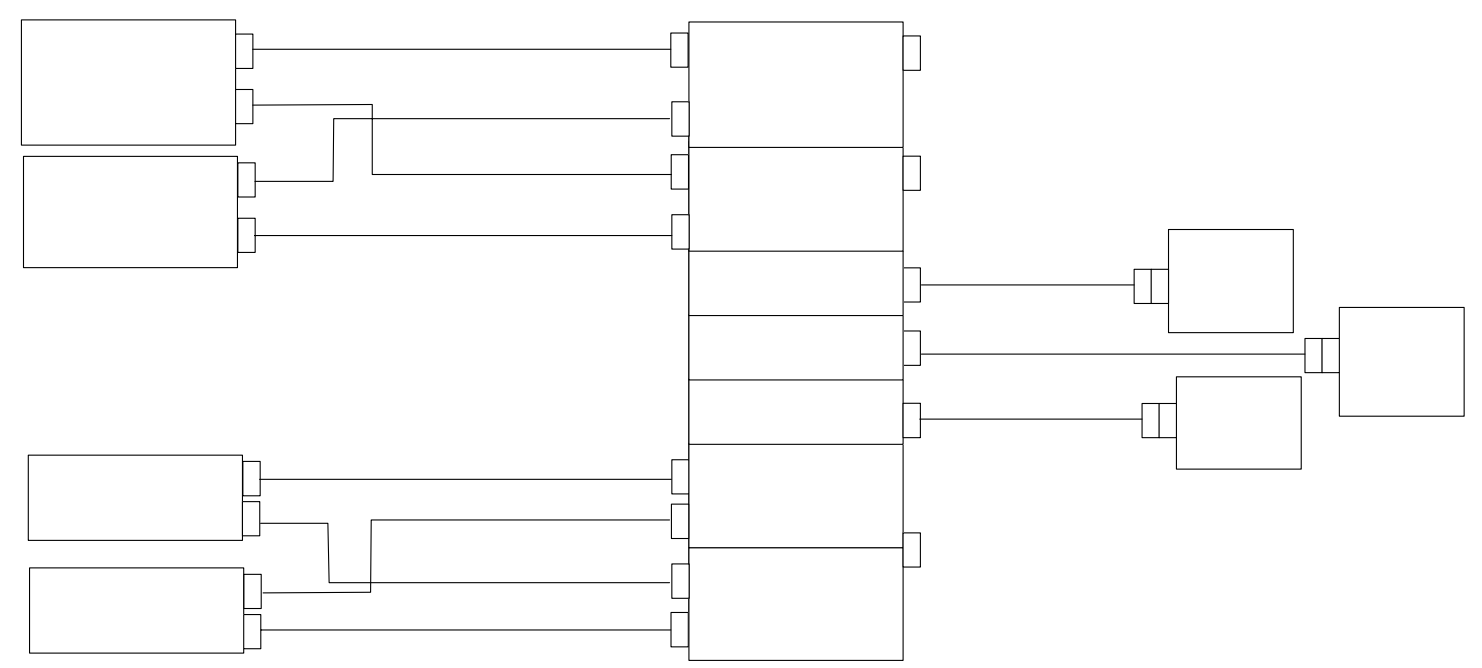

Figure 4. Block Diagram of Focal Plane System

software hierarchy. It communicates with the FPMs through a cryogenic harness and with the ICDH through a highspeed SpaceWire interface. The FPE receives its power from the spacecraft Electrical Power Unit (EPU). In turn, it provides the FPMs with their electrical power, bias control, and clocking to drive the detectors and readout electronics. It receives and digitizes the detector output signals- both science and housekeeping- and sends this data to the ICDH for 
storage and downlinking. The FPE also monitors and controls the temperature of each FPM and controls the detector annealing heaters.

\section{FPE Functional Requirements}

The FPE provides the following functions to facilitate the operation of the MIRI Focal Plane System:

- Subarray readout for a small number of fixed parameters

- Annealing and cool-down circuit capable of being command ON/OFF

- Temperature stability circuit set point command capability

- Selectable primary or redundant FPM heater elements

- Bias voltages set point

- Array readout intervals at discrete integration times, which are integral multiples of the detector frame time

- Enable and disable the reference output signal

- Select the reference to be used as an offset

- Power on and off the detector interface

- Enable clocking of detector after an exposure to ensure temperature stability

- Specify integration time as identified by the number of frames

- Pre-integration time frame reset speed and number

- Pixel processing mode of fast and slow

- Test pattern enable and disable

- Functionally redundancy at the circuit board level for the following functions:

- $\quad$ SpaceWire command and housekeeping telemetry I/F

- Power distribution

- $\quad$ Read out $>1 \mathrm{M}$ pixels per frame for each of three detectors

- Reading out the detector in $<3$ seconds.

The primary requirements on the cryogenic FPE-FPM harness is to provide a low noise environment, while transitioning from the 7.1 K FPM cryogenic temperature to the FPE's room temperature of $300 \mathrm{~K}$. The design of the harness minimizes the total heat load to the FPM, and thus to the dewar cryogen. There is one set of signal/control and temperature control/monitoring harnesses for each FPM. Having a overall length of approximately 4 meters, the harnesses are divided into a short, $\sim 1 \mathrm{~m}$, sections that are routed from their FPM to an instrument connector panel (ICP) located at the foot of one of OBA hexapod mounts. This is also the where the harness is thermally sunk at $37 \mathrm{~K}$ to the ISIM. The remaining 3 meters is used in the routing from the ICP over the ISIM structure to the FPE.

\section{THERMAL DESIGN}

The overall MIRI system, including the Dewar has been optimised for minimum mass and volume, which is achieved by using only a solid hydrogen cryogen in a two stage Dewar concept. As a consequence, to minimise complexity as well as mass, the overall Optical Bench Assembly of the MIRI adopted a single thermal stage design at $\sim 8-9 \mathrm{~K}$. This temperature is close to that necessary for detector operation, but is colder than necessary to minimise the mid-infrared thermal self-emission component of the optical noise background (15.5K). However, the benefits of adopting a single thermal stage in terms of mass, volume and overall simplicity are substantial, and so an overall thermal design concept with a negligible thermal self-emission in the mid-infrared results.

To minimise the temperature and maximise the thermal stability of the three detectors, they are thermally isolated from the Optics Module and connected directly to a separate solid hydrogen stage in the Dewar via a dedicated heat strap. This strap takes only the detector load thereby minimising the temperature difference between the detectors and the cryogen surface and the separate Dewar stage for detector cooling is optimised for the detector temperature requirements. A second heat strap connects the rest of the Optical Bench Assembly to the Dewar, taking the bulk of the Instrument thermal load where temperature and stability are not so critical. 
Another strong system mass driver is the total heat leak into the MIRI and consequently the dewar. An overall budget of $60 \mathrm{~mW}$ (including margin) has been set, along with a corresponding mass allocation for the dewar of $208 \mathrm{~kg}$. The significant contributors to this budget are the radiation load from the ISIM, the structural supports to the ISIM, and the conduction from the housekeeping and data harnesses. The radiation is generally minimised by the overall compact size of the Optical Bench assembly, and by a low emissivity MLI cover. The structural supports are optimally designed to minimise the thermal conduction, whilst meeting the required stiffnes. To this end, a Carbon-Fibre Reinforced Polymer six-strut design has been selected for the optimum ratio of stiffness to conductivity at these cryogenic temperatures. Harness conduction has been minimised by minimising the total number of mechanisms in the design overall (there are five mechanisms required to provide all the Instrument functionality) and paying close attention to the harness design to minimise conduction, taking appropriate redundancy considerations.

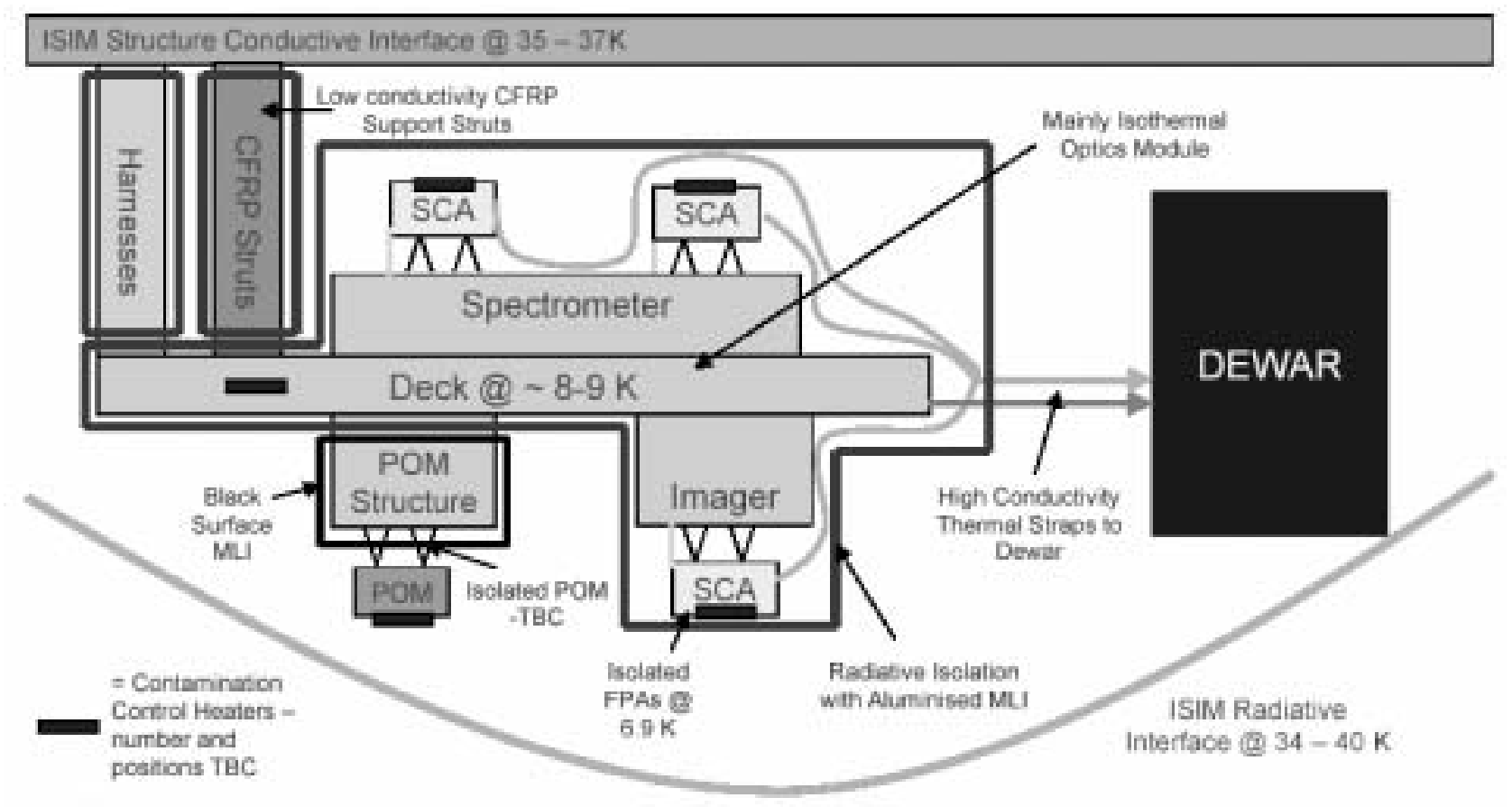

Figure 5 Thermal architecture

\subsection{Cooldown and Contamination Control}

The JWST is launched warm and then passively cooled on orbit with the ISIM reaching a final temperature of ca. 40K. Heat switches isolate the OBA from the Dewar until the ISIM is sufficiently cold. Details of the MIRI thermal design and the cool down process can be found in Heys et al. ${ }^{7}$

Current models of the outgassing of the JWST and ISIM structure indicate that when the ISIM reaches operating temperature $\sim 1.5 \mu \mathrm{m}$ of ice will have coated exposed surfaces. This is optically unacceptable. The JWST contamination control plan requires the cooldown of the instruments to lag significantly behind that of the ISIM in order to protect the optical surfaces. For MIRI the degree of lag possible is tied to the ISIM thermal environment details and the timing of the heat switch closure - once connected to the Dewar the MIRI optics and detectors rapidly become the coldest part of the system. In addition there are various contaminants which are still volatile at $40 \mathrm{~K}$ which will over time accumulate on exposed 7K MIRI surfaces. Based on the contamination studies to date we have therefore included a contamination control cover in the MIRI design. This cover is just inside the optical train after the pick-off mirror and closes off the instrument (there is no space in the ISIM in front of the POM). The POM is thermally isolated from the rest of the OBA 
structure and can be heated during the cooldown and heated to remove contaminants in flight. The cover will also be used to keep the optics and detectors clean during ground operation.

\section{STRUCTURAL CONCEPT}

The thermal isolation of the $7 \mathrm{~K}$ Instrument from the warmer ISIM $(\sim 35 \mathrm{~K})$ drives the Primary Structure concept to Struts whose function is minimising thermal conduction for a given stiffness and strength, and an Isothermal Deck, whose function is primarily meeting strength considerations at minimum mass. Secondary structures maintain alignment of optical components with minimum mass for each optical module.

The Primary Structure design incorporates a six strut, isostatic mount with end fittings that provide the degrees of freedom for kinematic mounting to the ISIM. At the other end, the strut hexapod interfaces via hard points to the Deck. With the exception of the filters and struts the Instrument is made entirely from 6061 (T6) Aluminium alloy resulting in a design that cools iso-elastically, thereby minimising internal thermo-elastic distortions to negligible levels compared to optical tolerances. Each of the optical sub-assemblies has a supporting structure for the mirrors, and this structure is hard-mounted to the Deck of the Primary Structure. The overall structure and packaging concept is shown in Figure 6. The design and first test results of the thermally isolating hexapod are described in Jessen et $\mathrm{al}^{8}$
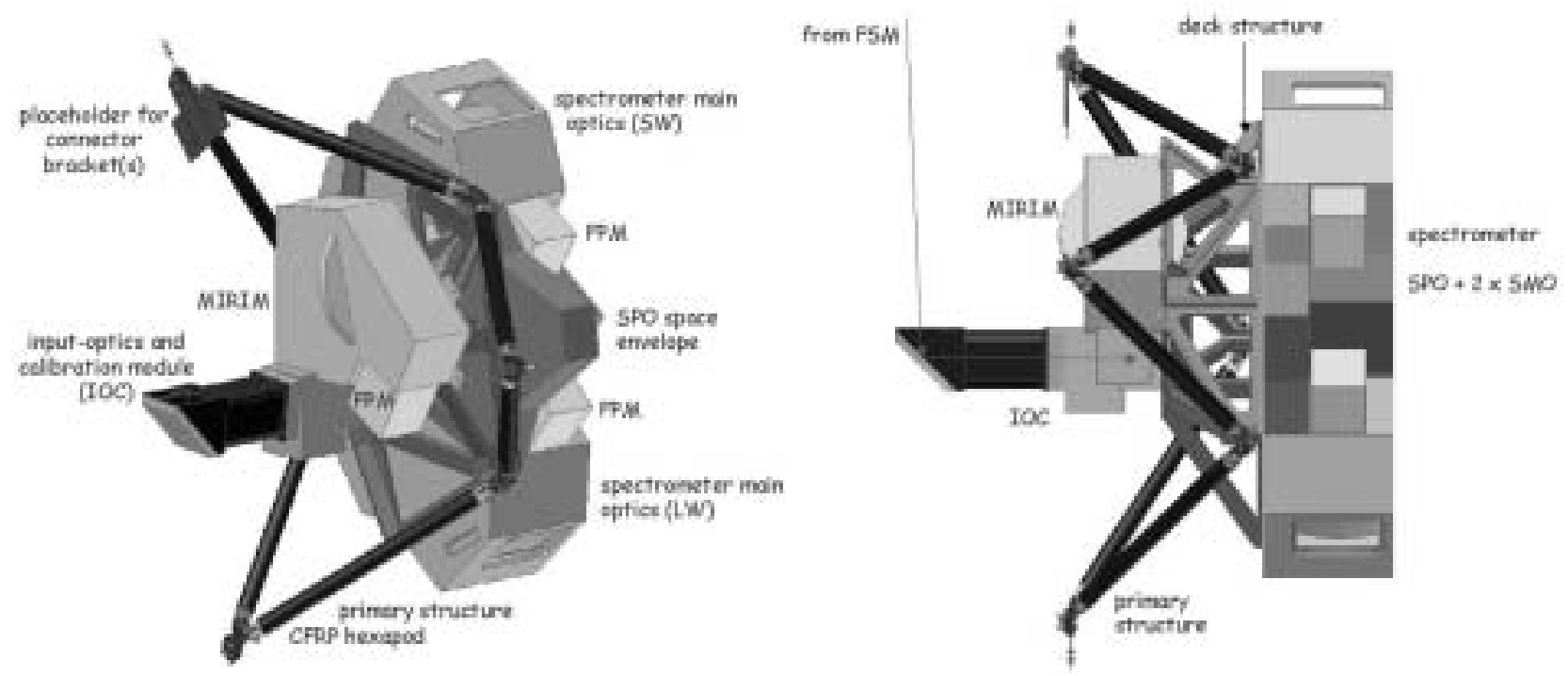

Figure 6 : Overall Structural Concept showing CFRP Hexapod and light-weighted Aluminium Deck

\section{MECHANISMS AND INSTRUMENT CONTROL ELECTRONICS.}

There are a total of 5 cryo-mechanisms in MIRI:

- A Contamination Control Cover with functions described above

- A filter wheel in the Imager module that also contains the pupil stops for the coronographs and a prism for low resolution spectroscopy

- Two identical wheels in the spectrometer each of which rotates to select both a dichroic and a corresponding grating.

- A small flip mirror which is an identical copy of a flip mirror used by the Herschel PACS instrument is used to direct calibration light into the spectrometer when needed 
It is intended that the imager wheel and spectrometer wheels will have sufficient similarity that a common qualification can be used. All of the wheel mechanisms have considerable heritage from ISO, and are at an advanced stage of development. Lemke et al ${ }^{9}$ describe these mechanisms in detail. The contamination control cover is the subject of competitive industrial studies at this time and so is not described further.

The motors and harness for the wheel mechanisms has been carefully optimized for low thermal impact in a cryogen cooled instrument. The mechanism motors are implemented in hot redundancy, specifically there are two systems of coils in each motor. Nominally both systems of coils are operated in series. In the event of a problem with one system of coils in a motor, this failed system of coils can be electrically isolated via relays in the ICE. In this case the motor can still be operated with slightly reduced efficiency. The prime and redundant coil systems utilise a common return, to minimise the amount of wiring and thereby heat conduction into the OBA. To improve the robustness of the cryoharness, each motor drive wire is duplicated in the harness (i.e. two wires are used between each pin-to-pin connection).

The motors do not draw power when not operating. The non-operating dissipation of the mechanisms is therefore zero. The heat dissipation when operating is also extremely low $(<0.2 \mathrm{~mJ}$ per step), resulting in a negligible impact of the overall heat-load budget.

The Instrument Control Electronics provides the capability to control the four mechanisms, process mechanism position sensor and thermistor telemetry, and drive the calibration emitters. The ICE is connected to the ISIM Command \& Data-Handling electronics by an IEEE-1355 bus, and to the ISIM PDU primary power supply. The ICE is located in the region2 part of the ISIM, and thus subject to a tight power requirement, and has harness links to the OBA provided by NASA GSFC. The ICE operates in cold redundancy, with A/B side switching controlled from the ISIM PDU.

\section{DEVELOPMENT PLAN AND SCHEDULE}

To meet the aggressive schedule for the JWST instruments, the MIRI development plan has been based around 3 models with the two early developments mitigating key risk areas. The Structure Thermal Model (SM/STM) provides an early mechanical qualification of the Primary Structure, and is used to characterise gravity release and alignment repeatability warm to cold $(77 \mathrm{~K})$, validate the thermal model, and de-risk the test facility prior to VM. The STM is later refurbished with additional mirrors and delivered to GSFC for use during the ISIM ETU tests in 2007. The Verification Model (VM) is used to verify some key aspects of instrument performance at operating temperature sufficiently early to avoid major cost and schedule problems in the event of detected problems requiring extensive FM modifications. It contains the flight optical design, but not all optics channels are fully populated, the DM mechanisms, and a complete Focal Plane System with flight-like detectors and software. Qualification of the remaining aspects happens on the (proto) Flight Model .

The MIRI programme is now well established and entering the development hardware construction phase. Construction of the STM model of MIRI will begin at the Rutherford Appleton Laboratories this August. A high level of design maturity has been achieved and the MIRI Preliminary Design Review will be held in January 2005. The instrument is on schedule for delivery to GSFC for integration into the ISIM in January 2009.

\section{ACKNOWLEDGEMENTS}

We would like to thank all the members of the MIRI team for their participation in this work.. The work described in this paper was supported by ESA, NASA and European Consortium National Funding Agencies in the UK, France, Germany, Switzerland, Netherlands, Denmark, Sweden, Belgium, Ireland and Spain.

\section{REFERENCES}

1. J.P. Gardener et al., "The science goals of the James Webb Space Telescope", Optical Infrared and Millimeter Space Telescopes, Proc. SPIE 5487 (this volume), 2004. 
2. B.M. Swinyard et al., "Sensitivity Estimates for the mid infrared instrument (MIRI) on the JWST", Optical Infrared and Millimeter Space Telescopes, Proc. SPIE 5487 (this volume), 2004.

3. P.S. Davila et al. "The James Webb Space Telescope Science Instrument Suite:an overview of optical designs", Optical Infrared and Millimeter Space Telescopes, Proc. SPIE 5487 (this volume), 2004.

4. M. Wells et al. "The design and fabrication of multiple dichroic beamsplitters for the MIRI spectrometer", Optical Infrared and Millimeter Space Telescopes, Proc. SPIE 5487 (this volume), 2004.

5. D. Lee et al. "Image Slicers Design for Manufacture", Optical Fabrication, Metrology, and Material Advancements for Telescopes, Proc. SPIE 5494, 2004.

6. P.J.Love et al. "1Kx1K Si:As IBC detector arrays for JWST MIRI and other applications" Optical and Infrared Detectors for Astronomy, Proc. SPIE 5499, 2004.

7. S.C.Heys et al "Thermal and Contamination Control of the mid-infrared instrument for JWST", Modeling and Systems Engineering for Astronomy, Proc. SPIE 5497, 2004.

8. N.C. Jessen et al. "The CFRP primary support structure for the MIRI instrument on board the James Webb Space Telescope", Astronomical Structures and Mechanisms Technology, Proc. SPIE 5495, 2004.

9. D. Lemke et al. "Positioning of the elements in the cryogenically cooled infrared instrument MIRI for the James Webb Space Telescope", Astronomical Structures and Mechanisms Technology, Proc. SPIE 5495, 2004. 